\title{
Automorphisms on complex simple Lie algebras of order 3
}

\author{
Ching-I Hsin* \\ Dept. of Multimedia and Game Development, Minghsin University of Science and Technology \\ Hsinchu County, Taiwan. \\ *Corresponding author: hsin@must.edu.tw
}

\begin{abstract}
For complex simple Lie algebras, the article provides the classification of all automorphisms of order 3. The method is an extension of Dynkin diagrams so that the classification is a listing of diagrams that represent automorphisms of order 3. This work extends an earlier result on automorphisms of order 2. As an application, it shows that for automorphisms of orders 2 and 3 only, the invariant subalgebra determines the automorphism.
\end{abstract}

2010 Mathematics Subject Classification: 17B20, 17B05

Keywords: Automorphism, Dynkin diagram, invariant subalgebra, Lie algebra.

\section{Introduction}

Let $\mathfrak{g}$ be a finite-dimensional complex simple Lie algebra (Humphreys, 1973). Let $\mathfrak{h}$ be a Cartan subalgebra of $\mathfrak{a}$, and let $\Delta \subset \mathfrak{h}^{*}$ be its root system. It leads to a root space decomposition

$$
\mathfrak{g}=\mathfrak{h}+\sum_{\Delta} \mathfrak{g}_{\alpha} .
$$

Let $\Pi \subset \Delta$ be a simple system, with the lowest root $\varphi$. Let $\mathrm{D}^{1}$ be the extended Dynkin diagram of $\mathfrak{g}$ so that its vertices are members of $\Pi \cup\{\varphi\}$. The roots $\Pi \cup\{\varphi\}$ are linearly dependent, and there are unique relatively prime positive integers $\left\{a_{\alpha}\right\}_{D^{1}}$ such that $\sum_{D^{1}} a_{\alpha} \alpha=0$ (Kac, 1990). Here relatively excellent means that they have no common factor other than 1.

A Kac diagram $C$ is an assignment of relatively prime nonnegative integers $\left\{C_{\alpha}\right\}_{D^{1}}$. Given a Kac diagram $c$, we let $n=\sum_{\mathrm{D}^{1}} a_{\alpha} c_{\alpha}$ and let $\omega_{n}=\exp (2 \pi \mathrm{i} / n) \in \mathrm{C}$. We say that $c$ represents a $\mathfrak{a}$ automorphism $\theta: \mathfrak{g} \rightarrow \mathfrak{g}$ if 


$$
\theta(X)=\left\{\begin{array}{cl}
X & \text { for } X \in \mathfrak{h}, \\
\omega^{c_{\alpha}} X & \text { for } X \in \mathfrak{g}_{\alpha} \text { and } \alpha \in \mathrm{D}^{1} .
\end{array}\right.
$$

In this case, $\theta$ has order $n$, because $n$ it is the smallest positive integer such that $\theta^{n}=1$.

Two $\mathfrak{g}$-automorphisms $\theta, \theta^{\prime}$ are said to be equivalent if there is a $\mathfrak{g}$-automorphism $\sigma$ such that $\theta^{\prime}=\sigma \theta \sigma^{-1}$. The list of all $\mathfrak{g}$-automorphisms of order two up to equivalence has been given (Chuah, 2012, Figure 1-3). Automorphisms of order 2 play essential roles in the study of real semisimple Lie algebras and symmetric spaces. This method is extended here to apply automorphisms of order 3 for generalized symmetric spaces, thereby establishing the importance of automorphisms of order 3 (Wolf \& Gray, 1968). In this article, we provide a diagrammatic classification of all $\mathfrak{g}$ automorphisms of order 3 .

The group of all $\mathfrak{g}$-automorphisms, denoted by aut( $\mathfrak{g})$, has a natural topology structure. We say that $\theta \in \operatorname{aut}(\mathfrak{g})$ is inner if it lies in the connected component, which contains the identity map. Otherwise, we say that $\theta$ is outer.

Theorem 1.1. Up to equivalence, all inner $\mathfrak{g}$-automorphisms of order three are represented by the Kac diagrams in Figure 1 for classical Lie algebras and Figure 2 for exceptional Lie algebras.

In these Kac diagrams, we make the convention that the vertices $\alpha$ such that $c_{\alpha}=0$ are drawn without indicating the integer 0 . For example, in the first diagram of Figure 2, two vertices satisfy $c_{\alpha}=1$, and five vertices satisfy $\boldsymbol{c}_{\alpha}=0$.

The complex simple Lie algebras are classified by their Dynkin diagrams, which are $A_{n}, B_{n}$, $C_{n}, D_{n}, E_{6}, E_{7}, E_{8}, F_{4}, G_{2}$ (see Section 11.4 of Humphreys, 1973). In particular, the Lie algebras of $A_{n}, B_{n}, C_{n}, D_{n}$ are constructed (see Section 1.2 of Humphreys, 1973).

There are more complicated diagrams, known as twisted diagrams. For the Lie algebra $D_{4}$, there is a twisted diagram $\mathrm{D}^{3}$, which we will explain later in (3).

Theorem 1.2. Up to equivalence, there are only two outer $\mathfrak{g}$-automorphisms of order 3. They occur when $\mathfrak{g}=D_{4}$ and are represented by the twisted diagrams in Figure 3.

The fixed points of mappings are studied in many areas of mathematics, see for example (Pant, Chauhan, Cho \& Gordji, 2015). We also study the fixed points here. Given a $\mathfrak{a}$-automorphism $\theta$, we let $\mathfrak{g}^{\theta}$ denote its fixed point set, also known as invariant subalgebra, namely

$$
\mathfrak{g}^{\theta}=\{X \in \mathfrak{x} ; \theta(X)=X\} \text {. }
$$

As an application of the lists of diagrams, we study to what extent $\mathfrak{a}^{\theta}$ determine $\theta$. It is known that for automorphisms of order 2 , the isomorphic class of $\mathfrak{a}^{\theta}$ determines the equivalence class of $\theta$. The following theorem says that this is also true for automorphisms of order 3 , but not higher order automorphisms. The theorem $\cong$ denotes Lie algebra isomorphism. 
Theorem 1.3. Let $\theta$, $\sigma$ be g-automorphisms of order $n$.

(a) For $n=2,3, \theta$ and $\sigma$ are equivalent if and only if $\mathfrak{a}^{\theta} \cong \mathfrak{a}^{\sigma}$.

(b) For $n \geq 4$, the condition, $\mathfrak{g}^{\theta} \cong \mathfrak{g}^{\sigma}$ does not imply that $\theta$ and $\sigma$ are equivalent.

We shall prove Theorems 1.1 and 1.2 in Section 2 and prove Theorem 1.3 in Section 3.

\section{Automorphisms of order 3}

Recall that $\mathfrak{x}$ is a finite-dimensional complex simple Lie algebra, $\mathfrak{h}$ is a Cartan subalgebra of $\mathfrak{x}, \Delta$ $\subset \mathfrak{h}^{*}$ is its root system, and

$$
\mathfrak{a}=\mathfrak{h}+\sum_{\Delta} \mathfrak{g}_{\alpha}
$$

is the root space decomposition.

We recall a result of Kac on finite order $\mathfrak{a}$-automorphisms. Let $\Pi \subset \Delta$ be a simple system. Let $\sigma \in \operatorname{aut}(\Delta)$ be a root automorphism of order $k$ which stabilizes $\Pi$, namely $\sigma(\Pi)=\Pi$. Clearly, $\sigma$ can be the identity. Otherwise, the list of Dynkin diagrams show that $\sigma \neq 1$ occurs only in the following cases,

$$
\begin{aligned}
k=1: & \text { any } \mathfrak{x}, \\
& \quad k=2: \mathfrak{g}=A_{n}(n \geq 2), D_{n}, E_{6}, \\
& k=3: \mathfrak{d}=D_{4} .
\end{aligned}
$$

Let $\mathbb{Z}_{\mathrm{k}}$ denote the group of integers modulo $k$. There exists a Lie algebra automorphism of order $k$, still denoted by $\sigma$, such that

$$
\sigma \mathfrak{g}_{\alpha}=\mathfrak{g}_{\sigma \alpha}
$$

for all $\alpha \in \Delta$. It leads to a $\mathbb{Z}_{\mathrm{k}}$-grading $\mathfrak{g}=\sum_{\mathbb{Z}_{\mathrm{k}}} \mathfrak{a}_{r}$, where $\mathfrak{a}_{r}$ has the eigenvalue $\exp (2 r \pi i / k)$ with respect to $\sigma$. Then $\mathfrak{q}_{0}$ is a subalgebra of $\mathfrak{a}$, and it acts on $\mathfrak{a}_{r}$ for all $r \in \mathbb{Z}_{\mathrm{k}}$. We construct a diagram.

$\mathrm{D}^{k}=$ set of vertices representing $\Pi_{0} \cup\{\varphi\}$,

where $\Pi_{0}$ is a simple system of $\mathfrak{q}_{0}$, and $\varphi$ is the lowest weight of the $\mathfrak{a}_{0}$-representation on $\mathfrak{a}_{1}$. Also, the edges of $\mathrm{D}^{k}$ are drawn according to the usual rule of Dynkin diagrams. Note that if $\sigma$ is the identity, so that $k=1$, then $\mathfrak{g}=\mathfrak{g}_{0}=\mathfrak{a}_{1}$, so $\varphi$ is just the lowest root of $\Pi=\Pi_{0}$. In this case, $\mathrm{D}^{1}$ is just the extension of the Dynkin diagram of $\mathfrak{g}$. 
Having added $\varphi$ to $\Pi_{0}$, it follows that the roots represented by the vertices of $\mathrm{D}^{k}$ are linearly dependent, so there are unique relatively prime positive integers $\left\{a_{\alpha}\right\}_{\mathrm{D}^{k}}$ such that

$$
\sum_{\mathrm{D}^{k}} a_{\alpha} \alpha=0 .
$$

These integers are presented in Chapter 4, Tables Aff 1, Aff 2, and Aff 3 (Kac, 1990).

A Kac diagram $c$ an assignment of relatively prime nonnegative integers to $\mathrm{D}^{k}$, namely $c_{\alpha}$ is a nonnegative integer for each $\alpha \in \mathrm{D}^{k}$, and $\left\{c_{\alpha}\right\}_{\mathrm{D}^{k}}$ is a set of relatively prime integers. Two Kac diagrams on $\mathrm{D}^{k}$ are said to be equivalent if they are related by a diagram symmetry of $\mathrm{D}^{k}$. If $n$ is a positive integer, we let $\omega_{n}=\exp (2 \pi i / n) \in \mathrm{C}$ be the primitive $n$-th root of unity.

Theorem 2.1. (Kac) (Helgason, 2001, Chapter X; Kac, 1990, Chapter 8) There is a bijective correspondence between the equivalence classes of Kac diagrams and finite order $\mathfrak{a}$ automorphisms. In this correspondence, if $c$ is a Kac diagram, it represents a $\mathfrak{a}$-automorphism $\theta$ of order $n=k \sum_{\mathrm{D}^{k}} a_{\alpha} c_{\alpha}$, where there exist root vectors $\left\{X_{\alpha}\right\}_{\mathrm{D}^{\mathrm{k}}}$ such that $\theta\left(X_{\alpha}\right)=\omega_{n}^{c_{\alpha}} X_{\alpha}$. Here $\mathfrak{u}^{\theta}$ is reductive, its semisimple part has a Dynkin diagram $\left\{\alpha \in \mathrm{D}^{\mathrm{k}} ; c_{\alpha}=0\right\}$, and its center has dimension.

$\#\left\{\alpha \in \mathrm{D}^{\mathrm{k}} ; c_{\alpha} \neq 0\right\}-1$.

The case where $n=2$ has been classified in (Chuah, 2012, Figures 1-3). In this article, we classify the Kac diagrams $n=3$ corresponding to all $\mathfrak{a}$-automorphisms of order 3 . By Theorem 2.1, we look for all Kac diagrams $c$ such that

$$
k \sum_{D^{\mathrm{k}}} a_{\alpha} c_{\alpha}=3 .
$$

Recall that $k$ can be 1,2,3 as discussed in (2). The condition (4) excludes $k=2$, so we deal with two cases,

(a) $k=1$ for any,

(b) $k=3$ for $\mathfrak{a}=D_{4}$.

We start with (5)(a). Here (4) becomes $\sum_{D^{1}} a_{\alpha} c_{\alpha}=3$, which leads to the following four cases:

(a) $a_{\alpha}=c_{\alpha}=a_{\beta}=c_{\beta}=a_{\gamma}=c_{\gamma}=1$,

$$
c=0 \text { on } \mathrm{D}^{1} \backslash\{\alpha, \beta, \gamma\} \text {, }
$$


(b) $a_{\alpha}=a_{\beta}=1, c_{\alpha}=1, c_{\beta}=2, c=0$ on $\mathrm{D}^{1} \backslash\{\alpha, \beta\}$,

(c) $a_{\alpha}=1, a_{\beta}=2, c_{\alpha}=c_{\beta}=1$,

$c=0$ on $\mathrm{D}^{1} \backslash\{\alpha, \beta\}$,

(d) $a_{\alpha}=3, c_{\alpha}=1, c=0$ on $\mathrm{D}^{1} \backslash\{\alpha\}$.

We make the convention that for the Kac diagrams $c$ in Figures 1-3, the vertices $\alpha$ without indicated integer refer to $c_{\alpha}=0$. Figures 1-2 provide all Kac diagrams which satisfy one of the conditions of (6). For example:

the first Kac diagram of $A_{n}$ satisfies (6)(a),

the second Kac diagram of $A_{n}$ satisfies (6)(b),

the first Kac diagram of $B_{n}$ satisfies (6)(c),

the second Kac diagram of $G_{2}$ satisfies (6)(d).

We conclude that Figures 1-2 classifies all Kac diagrams with $k=1$. Figure 1 deals with the classical Lie algebras $A_{n}, B_{n}, C_{n}, D_{n}$, and Figure 2 deals with the exceptional Lie algebras $E_{6}, E_{7}$, $E_{8}, F_{4}, G_{2}$.

Next, we consider (5)(b). In this case, $\mathfrak{g}=D_{4}$ and (4) becomes $\sum_{\mathrm{D}^{3}} a_{\alpha} c_{\alpha}=1$. So there is exactly one vertex $a_{\alpha}=c_{\alpha}=1$, and $c$ assigns 0 to the remaining vertices of $\mathrm{D}^{3}$. Such Kac diagrams are classified by Figure 3.

We conclude that, by Theorem 2.1, Figures 1-3 classify all the Kac diagrams for $\mathfrak{x}$ automorphisms of order 3.

Recall that a $\mathfrak{g}$-automorphism is called inner if it lies in the connected component of aut(a) which contains the identity mapping and is called outer otherwise. In this diagrammatic classification, the automorphism is inner if and only if the diagram is $\mathrm{D}^{1}$ (Chuah, 2012, (4.4)). Therefore, the automorphisms represented by Figures 1-2 are inner, and the automorphisms represented by Figure 3 are outer. This completes the proof for Theorems 1.1 and 1.2.

\section{Invariant subalgebras}

Given a Kac diagram $c$ on $\mathrm{D}^{k}$, we define its kernel as the subdiagram of $\mathrm{D}^{k}$ provided by

$$
\operatorname{ker} c=\left\{\alpha \in \mathrm{D}^{k} ; c_{\alpha}=0\right\}
$$

In the following proposition, isomorphism of diagrams is understood as bijection of vertices which preserves edge relations. Let $\not \neq$ denote diagrams that are not isomorphic.

Proposition 3.1. Let $c$, $c^{\prime}$ be Kac diagrams on $\mathrm{D}^{k}$, which represent $\mathfrak{a}$-automorphisms of order 3. 
If $c, c^{\prime}$ are not equivalent, then $\mathrm{ker} c \not$ ker $c^{\prime}$.

Proof. This is proved by checking through the list of Kac diagrams in Figures 1-3. For example, consider the five Kac diagrams of $\mathfrak{g}=E_{6}$ in Figure 2. Their kernels are respectively the Dynkin diagrams of $A_{5}, A_{1}+A_{4}, D_{5}, A_{2}+A_{2}+A_{2}, D_{4}$, so these kernels are not isomorphic to one another. Similarly, for $\mathfrak{g}=E_{7}$, Figure 2 provides five Kac diagrams whose kernels are respectively the Dynkin diagrams of $D_{6}, A_{1}+D_{5}, A_{6}, A_{2}+A_{5}, E_{6}$, so these kernels are not isomorphic to one another.

By checking through all the Kac diagrams in Figures 1-3, Proposition 3.1 follows.

We shall see that Proposition 3.1 fails for automorphisms of order $\geq 4$, as Figure 4 provides a counterexample.

Recall that, for a $\mathfrak{g}$-automorphism $\theta$, we let $\mathfrak{a}^{\theta}$ denote its invariant subalgebra (1). We now prove Theorem 1.3(a). Let $\theta, \sigma$ be $\mathfrak{a}$-automorphisms. If they are equivalent, then there exists a $\mathfrak{a}$ automorphism $\mu$ such that $\sigma=\mu \theta \mu^{-1}$. It implies that $\mu\left(\mathfrak{a}^{\theta}\right)=\mathfrak{a}^{\sigma}$, hence $\mathfrak{g}^{\theta} \cong \mathfrak{g}^{\sigma}$. So the remaining issue is to prove the "if" part of the statement. The a-automorphisms of order 2 are also known as involutions. The result for involutions is known in the literature nevertheless, we elaborate the argument here. There is a bijective correspondence between the equivalence classes of $\mathfrak{a}$ involutions $\theta$ and isomorphic classes of real forms $\mathfrak{g}_{\mathrm{R}}$ of $\mathfrak{g}$, given by $\theta$ stabilizes $\mathfrak{a}_{\mathbb{R}}$ and is a Cartan involution of $\mathfrak{q}_{\mathrm{R}}$. See, for instance (Chuah, 2012, §2). Let $\theta$, $\sigma$ be $\mathfrak{a}$-involutions. They correspond to some real forms $\mathfrak{g}_{\mathbb{R}}, \mathfrak{a}^{\prime}{ }_{\mathrm{R}}$ of $\mathfrak{g}$. If $\mathfrak{a}^{\theta} \cong \mathfrak{g}^{\sigma}$, then $\mathfrak{a}_{\mathbb{R}} \cong \mathfrak{g}^{\prime}{ }_{\mathrm{R}}$ (Helgason, 2001, Chapter X-6, Theorem 6.2) and hence $\theta, \sigma$ are equivalent. This proves Theorem 1.3(a) for involutions.

Next, we prove Theorem 1.3(a) for $\mathfrak{g}$-automorphisms $\theta$, $\sigma$ of order 3. Theorems 1.1 and 1.2 are represented by Kac diagrams $c, c^{\prime}$ in Figures 1, 2, or 3. Suppose that $\theta, \sigma$ are not equivalent to each other. By Theorem 2.1, $c, c^{\prime}$ are not equivalent. By Proposition 3.1, ker $c ¥ \operatorname{ker} c{ }^{\prime}$. By Theorem 2.1, $\operatorname{ker} c, \operatorname{ker} c^{\prime}$ are the Dynkin diagrams of the semisimple parts of $\mathfrak{g}^{\theta}, \mathfrak{x}^{\sigma}$, so we have $\mathfrak{a}^{\theta} ¥ \mathfrak{g}^{\sigma}$. This proves Theorem 1.3(a) for automorphisms of order 3 .

Next, we prove Theorem 1.3(b). Let $n \geq 4$. It suffices to construct counterexamples of non-equivalent $\mathfrak{a}$-automorphisms $\theta$, $\sigma$ of order $n$ such that $\mathfrak{g}^{\theta} \cong \mathfrak{g}^{\sigma}$. Let $\mathfrak{g}=A_{m}$, where $m \geq 3$. Consider the two Kac diagrams in Figure 4. They are not equivalent, but their kernels are the same, namely the Dynkin diagram of $A_{m^{-2}{ }}$.

Suppose that $\theta, \sigma$ is represented by the Kac diagrams in Figure 4. By Theorem 2.1, $\theta$ and $\sigma$ are not equivalent because their Kac diagrams are not equivalent, but both their invariant subalgebras are $\mathfrak{x}^{\theta}=\mathfrak{g}^{\sigma}=A_{m^{-2}}+C^{2}$. With this counterexample, we have proved Theorem 1.3(b). 


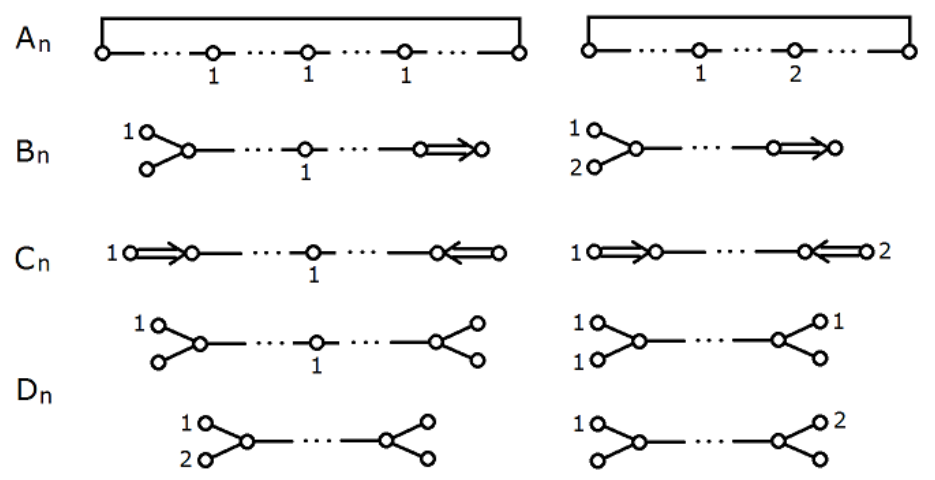

Fig. 1. Inner classical Kac diagrams of order 3
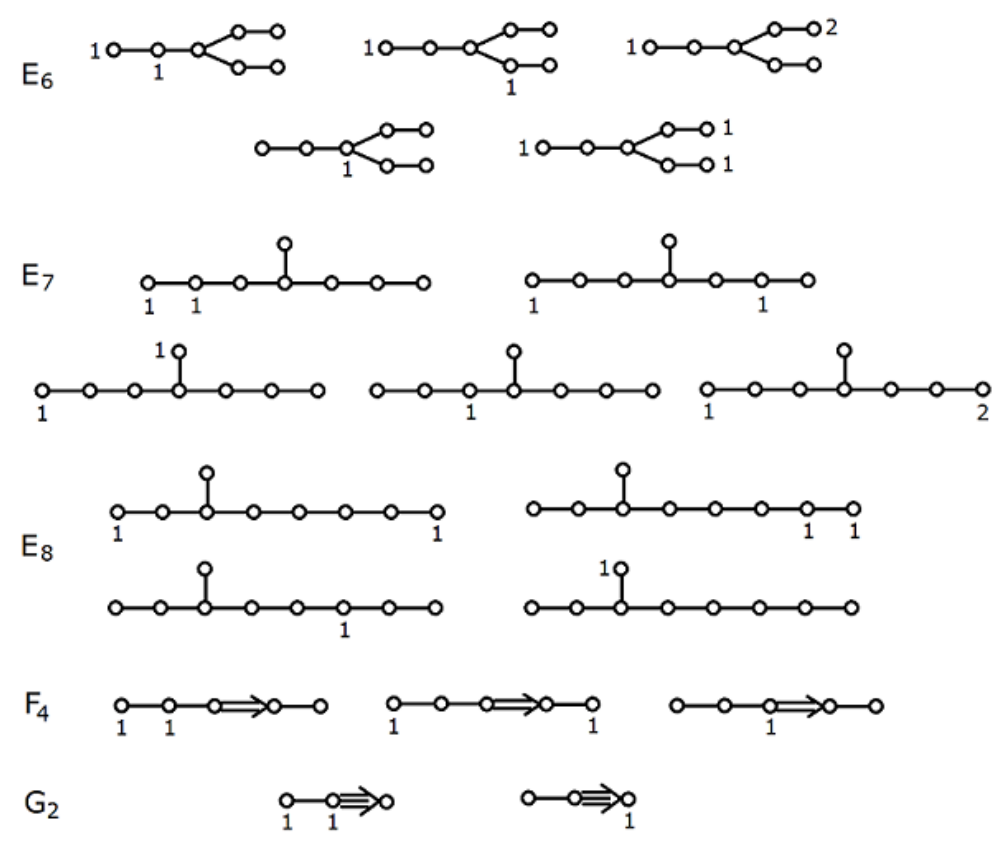

Fig. 2. Exceptional Kac diagrams of order 3.

$D_{4}$

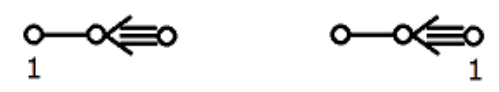

Fig. 3. Outer Kac diagrams of order 3.

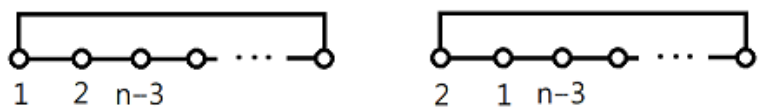

Fig. 4. Non-equivalent Kac diagrams with the same kernel. 


\section{References}

Chuah, M. K. (2012) Finite order automorphisms on real simple Lie algebras, Transactions of the American Mathematical Society 364: 3715-3749.

Helgason, S. (2001) Differential Geometry, Lie Groups, and Symmetric Spaces, Graduate Studies in Mathematics Vol. 34, American Mathematical Society, Providence.

Humphreys, J. E. (1973) Introduction to Lie algebras and representation theory, Graduate Texts in Mathematics Vol. 9, Springer.

Kac, V. G. (1990) Infinite Dimensional Lie Algebras, third edition, Cambridge University Press, Cambridge.

Pant, B. D., Chauhan, S., Cho, Y. J. \& Gordji, M. E. (2015) Fixed points of weakly compatible mappings in fuzzy metric spaces, Kuwait Journal of Science 42(2): 107-127.

Wolf, J. \& Gray, A. (1968) Homogeneous space defined by Lie group automorphisms, I and II, Journal of Differential Geometry 2: 77-159

Submitted: $\quad 02 / 10 / 2020$

Revised: $\quad 16 / 04 / 2021$

Accepted: $\quad 18 / 04 / 2021$

DOI: $\quad 10.48129 / \mathrm{kjs} .10668$ 